\title{
Editorial Introduction to Special Issue on Religion in Robotics
}

\author{
Gabriele Trovato $^{1} \cdot$ Yueh-Hsuan Weng $^{2} \cdot$ Antonio Sgorbissa $^{3} \cdot$ Rainer Wieching ${ }^{4}$
}

Accepted: 23 June 2021 / Published online: 2 July 2021

(c) The Author(s), under exclusive licence to Springer Nature B.V. 2021

In our world, religion and robotics may be regarded as distinct areas of human activity. In fact, they are not only deemed distinct, but also oppositional: it is widely acknowledged that here is little room for "the divine" in the realm of scientific investigation and technological development, and robotics is not considered an exception. However, in some ways, this intuitive categorization appears to be stereotyped and superficial. Generally speaking, it is straightforward to observe that, if one focuses on the study of religion and its impact on human cognition and societies as addressed by anthropologists, social scientists, psychologists, cognitive scientists or other human-centered research disciplines, the opposition with robotics research is only apparent. All these disciplines obviously share with robotics (and with research and technological development in general) a sound experimental approach, which is based on the formulation of hypotheses, the collection of data, and then the statistical analysis of such data to verify if hypothesis shall be accepted or rejected. A-priori opposition towards the convergence of religions and robotics ignores the past, the present and possibly the future of the multiple, non-obvious connections that they share.

The past teaches us that both religion and robotics share the objective of understanding humans and-to some extent - the dream of building an artificial human. There is evidence of such archetypes in many ancient cultures in the

\footnotetext{
Gabriele Trovato

gabriele@takanishi.mech.waseda.ac.jp

Yueh-Hsuan Weng

y.weng@srd.mech.tohoku.ac.jp

Antonio Sgorbissa

antonio.sgorbissa@unige.it

Rainer Wieching

rainer.wieching@uni-siegen.de

Waseda University, Tokyo, Japan

2 Tohoku University, Sendai, Japan

3 University of Genoa, Genoa, Italy

4 Siegen University, Siegen, Germany
}

world, from Greece to China. These representations have had positive as well as negative connotations, with differences normally relating to the specific cultural and religious background of the civilizations. More recently in the human history, questions about the nature of "conscience" and "free will" have been equally addressed by theologians and philosophers of mind, and in the last 50 years have become subject of investigation by AI and robotics experts as well, giving birth to a wide corpus of literature. This shows that, if the explanations provided by theology and computational cognitive models are likely not to be the same, they indeed may share somehow overlapping objectives.

Looking to the future from a pragmatic perspective, robots are expected to operate in contact with humans. Therefore, achieving fully autonomous human-robot interaction raises many issues that are typical of human-human interaction: the recent interest in Social Robotics about the role of "trust" in human-robot relationships, the quest for embedding ethics in robots and autonomous machines, the theories about robot emotions and the role of affective human-robot communication, the attempt to replicate Theory of Mind in robotics are only some examples. Moving further, as hybrid "communities" composed by humans and robots are expected to emerge in the close future, these issues are expected to acquire even a greater importance. Some countries, such as Japan, through the newly established Japanese Moonshot R\&D Program, have started realising the importance of religion for a role as a cultural basis to support the social system design of ELSI (Ethics, Legal, Social Issues) for AI-Robots Co-evolution towards 2050. Moreover, the recent discussion about granting a legal persona to robots, and the related consequences in terms of "robot rights" and "responsibilities", is an indicator of how the whole scientific community needs to be ready to address issues that, up to a few years ago, where only explored by ethicists or "unconventional" AI and robotics scientists. In order to be accepted, shall social robots of the future be aware of the spiritual beliefs of the persons they are interacting with? Shall they share (or pretend to share) these beliefs? On the other hand, we can foresee the automatisation of 
religion happening. After all, religions have changed due to the influence of emerging technologies (the printing press, the television, internet), and with AI and robotics on the horizon, we can expect this to happen again.

As to the present, the Covid-19 pandemic casts a long shadow over this question. The situation of social distancing and lockdown has highlighted new social needs; for instance, the need of older adults to be assisted and not to suffer social isolation and the possibility for believers to safely attend religious services. The field of robotics was caught by surprise by the pandemic, but it is expected to play an important role, not only in supporting human's daily work on pandemic control and prevention, such as disinfection, delivery of medications and food, measurement of vital signs, but also with social relations, helping to support communities psychologically and spiritually. In this regard, the few pioneering robots in this field, such as the Buddhist Xian'er, DarumaTO and Mindar, and the Christian BlessU-2 and SanTO, are starting to show their potential. As an aside, it shall be reminded that spiritual well-being is one of the aspects that, together with mental health, social participation and the reduction of social isolation and loneliness (among the others) is acknowledged as playing a key role in boosting health-related quality of life. This is also mentioned in WHO's definition of palliative care, namely: "an approach that improves the quality of life of patients and their families facing the problem associated with life-threatening illness, through the prevention and relief of suffering by means of early identification and impeccable assessment and treatment of pain and other problems, physical, psychosocial and spiritual." As such, spiritual well-being is subject to quantitative investigation through validated tools, such as the Functional Assessment of Chronic Illness Therapy-Spiritual Well-Being (FACIT-Sp-12) - a 12-item questionnaire that examines the relationships among spiritual well-being and health assessing three domains: Meaning, Peace and Faith.

This special issue covers this stimulating and potentially controversial topic. It originated in the 9th International Conference on Social Robotics (ICSR), held in Tsukuba, Japan. There, the "Religion in Robotics" workshop, organized by Gabriele Trovato, Francisco Cuellar and Alexander Huerta-Mercado, was the first of its kind. The workshop set out to "establish religion as a sub-field of Culturally-aware Robotics, by defining its theoretical background and its most relevant goals in today's world". The workshop focused also on cultures, aesthetics, RoboEthics, transhumanism, and robotics-related anthropology. Although initially a small niche, interest has eventually grown, given the rapidly increasing press coverage on the existing robots, and the successful workshop on "Robots and Religious Contexts", organized by Simon Balle and Charles Melvin Ess in RoboPhilosophy 2020.

For this issue, we originally selected five papers specifically on the topic of religion and robotics. However, due to several circumstances, two of them have been published separately, and will not be included in this issue, but only linked. Instead, a few other papers in closely related fields, will be joined..

The first selected one, is a review paper. It discusses the historical perspectives on the development of the relationship between religion and robotics; extends the focus on the contemporary societies, in their socio-cultural norms and values related to perception of robots in different cultures; and concludes with some predictions regarding a future of co-existence and co-dependence.

It is currently available in the April 2021 issue through this link: https://link.springer.com/article/10.1007/ s12369-020-00636-x.

The second paper we selected is the first one of this issue. It summarises the state of the art in robotics and religion. It also proposes a taxonomy for robot morphology that takes into account religion, and explains the novel concept of "theomorphic robots".

In the third paper (2nd in this issue) the author examines the relationship between human rights and the design of intelligent robots, in particular the potential application of the Universal Declaration of Human Rights to robots, discussing the concept of dignity and the "Moral Belief State".

The fourth paper focuses on Taoism to discuss the relationship between AI/robots, humans and gods, involving awareness and immortality. It is available in the December 2019 issue through this link:

https://link.springer.com/article/10.1007/ s12369-019-00594-z.

In the last paper of this special issue, the authors empirically investigate the user experience, acceptability and design features of social robots using the example of a Christian Protestant blessing ritual, based on the Christian robot BlessU2.

We hope that this special issue can lead people's interest on investigating various possibilities from an interdisciplinary approach to the study of religion and robotics.

Publisher's Note Springer Nature remains neutral with regard to jurisdictional claims in published maps and institutional affiliations. 